21st Particles and Nuclei International Conference (PANIC 2017)

International Journal of Modern Physics: Conference Series

Vol. 46 (2018) 1860070 (6 pages)

(C) The Author(s)

DOI: $10.1142 / \mathrm{S} 2010194518600704$

\title{
Tests of Lepton Flavour Universality at LHCb
}

\author{
Anna Lupato (On Behalf of the LHCb Collaboration) \\ University of Padua, Galileo Galilei \\ Via Marzolo 8, Padova (PD), 35131, Italy \\ anna.lupato@pd.infn.it
}

Published 3 May 2018

\begin{abstract}
In the Standard Model the electroweak coupling of the gauge bosons to leptons is independent of the lepton flavour. Semileptonic and rare decays of b quarks provide an ideal laboratory to test this property. Any violation of Lepton Flavour Universality would be a clear sign of physics beyond the Standard Model. In this work a review of the Lepton Flavour Universality tests performed using data collected by the LHCb experiment in 2011 and 2012 at a centre of mass energy of 7 and $8 \mathrm{TeV}$ is presented.
\end{abstract}

Keywords: Semileptonic decays; rare decays; lepton flavour violation.

\section{Introduction}

Lepton Flavour Universality (LFU) implies the equality of the coupling between the gauge bosons and the three families of leptons. It entails that the branching fractions of decays involving leptons do not depend on lepton flavour but differ only by phase space and helicity-suppressed contributions. Semileptonic and rare decays of heavy hadrons are an optimal laboratory to test LFU, since they allow to access all three generations of leptons. LFU is a Standard Model (SM) property by construction and therefore, any violation would be a clear sign of New Physics. Over the years, LFU has been tested and has proven to be accurate in several systems providing very strong limits. A large class of models extending the SM contains additional interactions involving enhanced couplings to the third lepton generation that could violate LFU. An extended Higgs Sector could have a large effect on semitauonic decay rates through the coupling of new charged Higgs scalars. ${ }^{1}$ Therefore, semileptonic decays of $b$ hadrons to the third generation provide a sensitive probe for such effects. In addition, also $b \rightarrow$ sll transitions constitute a good LFU probe since such processes are forbidden at tree level and the presence of unobserved particles entering in the

This is an Open Access article published by World Scientific Publishing Company. It is distributed under the terms of the Creative Commons Attribution 4.0 (CC-BY) License. Further distribution of this work is permitted, provided the original work is properly cited. 


\section{A. Lupato}

loops, like leptoquarks ${ }^{2,3}$ or Z', ${ }^{4,5}$ could alter their branching fractions. Ratios of branching fractions represent a powerful test of LFU since theoretical uncertainites cancel in the predictions and experimental systematics are much reduced. In the following sections, the results obtained analysing the LHC Run 1 data collected by LHCb experiment in 2011 and 2012 at a centre of mass energy of 7 and $8 \mathrm{TeV}$ are presented.

\section{LFU tests in $b \rightarrow$ sll transitions}

The comparison of the branching fractions of $B \rightarrow H \mu^{+} \mu^{-}$and of $B \rightarrow H e^{+} e^{-}$ decays allows precise test of LFU:

$$
R_{H}=\frac{\int_{q_{\min }^{2}}^{q_{\max }^{2}} \frac{d \Gamma\left(B \rightarrow H \mu^{+} \mu^{-}\right)}{d q^{2}} d q^{2}}{\int_{q_{\min }^{2}}^{q_{\max }^{2}} \frac{d \Gamma\left(B \rightarrow H e^{+} e^{-}\right)}{d q^{2}} d q^{2}}
$$

where $H$ represents a hadron containing a $s$ quark, such as $K$ or $K^{*}$ meson and the decay rate is integrated over a range of the squared dilepton invariant mass, $q^{2}$. This ratio is expected to be close to unity in the SM. ${ }^{6,7}$ Using the Run 1 data, $\mathrm{LHCb}$ has performed the measurement of the $R_{K}$ and $R_{K}^{*}$ ratios.

Both analyses measured the ratio

$$
R_{H}=\frac{\mathcal{B}\left(B \rightarrow H \mu^{-} \mu^{+}\right)}{\mathcal{B}\left(B \rightarrow H J / \psi\left(\rightarrow \mu^{-} \mu^{+}\right)\right)} / \frac{\mathcal{B}\left(B \rightarrow H e^{-} e^{+}\right)}{\mathcal{B}\left(B \rightarrow H J / \psi\left(\rightarrow e^{-} e^{+}\right)\right)}
$$

where $H=\left\{K, K^{* 0}\right\}$ and $K^{* 0} \rightarrow K^{+} \pi^{-}$. The candidates for the normalization channel $B \rightarrow H J / \psi\left(\rightarrow l^{-} l^{+}\right)$are selected using similar criteria to that of the nonresonant counterpart.

The first challenge of these measurements derives from the way muons and electrons interact with the detector. In particular, the former are characterized by a high reconstruction efficiency and clear experimental signature, while the latter emits a large amount of bremsstrahlung that results in a degraded $B$ momentum and mass resolution. Therefore, a bremsstrahlung recovery algorithm is used to improve the electron momentum reconstruction: clusters do not associated with charged tracks are searched in the electromagnetic calorimeter in a region defined by the extrapolation of the electron track upstream of the magnet and added to the measured electron momentum. This procedure is limited due to their energy threshold on the bremsstrahlung reconstructed photon $\left(\mathrm{E}_{T}>75 \mathrm{MeV}\right)$, the acceptance of the calorimeters and the presence of energy deposits wrongly interpreted as the bremsstrahlung clusters.

Moreover, due to the higher occupancy of the electromagnetic calorimeter compared to the muon stations, the constraint on the trigger bandwidth requires to impose higher thresholds on the transverse energy of electrons than those on the transverse momentum of muons, causing a partial loss of electron sample signal. To 

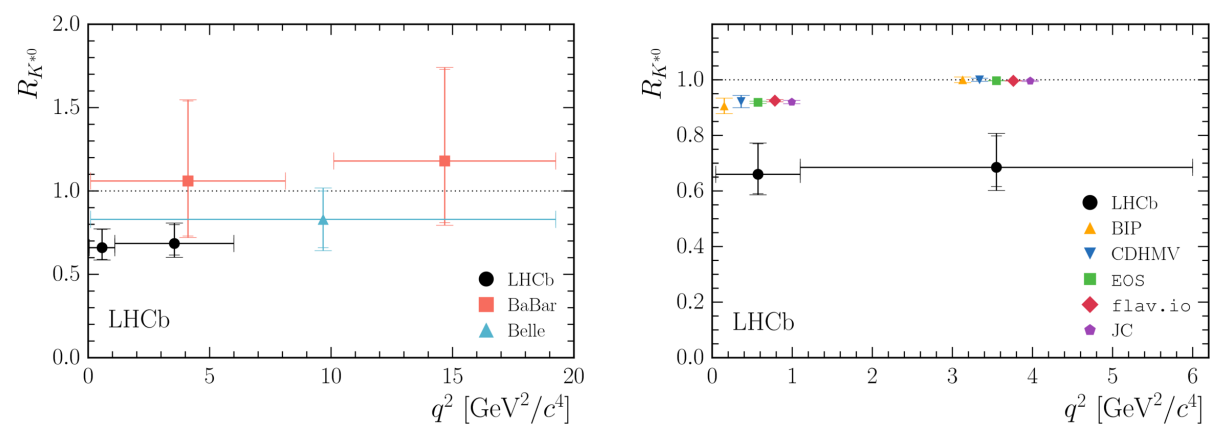

Fig. 1. Comparison of the $R_{K^{* 0}}$ with the previous results from the B-factories (left) and with SM expectations (right).

partially mitigate this effect, electron decays have been selected through the electron hardware trigger, using clusters associated to the kaon or $K^{* 0}$ decay products and selecting events that are not associated with B-decay products.

In 2014, the $\mathrm{LHCb}$ collaboration measured the $R_{K}$ ratio in the range $1<q^{2}<6$ $\mathrm{GeV}^{2} / c^{4}$ to be $0.745_{-0.074}^{+0.090}$ (stat) \pm 0.036 (syst), resulting in a $2.6 \sigma$ compatibility with the SM predictions. ${ }^{8}$

More recently, LHCb performed the measurement of the $R_{K^{*}}$ ratio. The measurement is performed in two $q^{2}$ bins that are sensitive to different physics contributions: the first one, $0.045<\mathrm{q}^{2}<1.1 \mathrm{GeV}^{2} / c^{4}$, dominated by the photon pole and the central bin, $1.1<\mathrm{q}^{2}<6 \mathrm{GeV}^{2} / c^{4}$, where the contribution from the $C_{9}^{\left({ }^{\circ}\right)}$ Wilson coefficient becomes more important.

Signal yields are extracted using an extended maximum likelihood fit to the reconstructed $B$ mass, simultaneously on three trigger categories and with the corresponding resonant mode counterpart. The ratio is measured to be

$$
R_{K^{* 0}}= \begin{cases}0.66_{-0.07}^{+0.11} \text { (stat) } \pm 0.03 \text { (syst) } & \text { for } 0.045<q^{2}<1.1 \mathrm{GeV}^{2} c^{4} \\ 0.69_{-0.07}^{+0.11} \text { (stat) } \pm 0.05 \text { (syst) } & \text { for } 1.1<q^{2}<6.0 \mathrm{GeV}^{2} c^{4}\end{cases}
$$

where the precision of measurement is limited by the size of the electron sample. The results are compatible with the SM expectations at the level of 2.1-2.3 $\sigma$ (low bin) and 2.4-2.5 $\sigma$ (central bin). Fig. 1 shows the comparison with previous $R_{K * 0}$ measurements from the B-factories and with theoretical expectations.

\subsection{LFU tests in $b \rightarrow$ clv transitions}

A precise measurement of a $B$ decay involving $\tau$ leptons is a challenge at an hadron collider due to the large background from partially reconstructed B hadron decays that mimic the signal topology. Moreover, the signal decay kinematics can not be fully constrained because of the neutrinos in the final state. In $2015, \mathrm{LHCb}$ has measured the branching fraction ratio $R_{D^{*}} \equiv \mathcal{B}\left(\bar{B}^{0} \rightarrow D^{*+} \tau^{-} \bar{\nu}_{\tau}\right) / \mathcal{B}\left(\bar{B}^{0} \rightarrow\right.$ 


\section{A. Lupato}

$\left.D^{*+} \mu^{-} \bar{\nu}_{\mu}\right)$ using a Run 1 sample. Only muonic tau decays, $\tau^{-} \rightarrow \mu^{-} \bar{\nu}_{\mu} \nu_{\tau}$ have been considered. The ratio is measured to be $R_{D^{*}}=0.336 \pm 0.027$ (stat) \pm 0.030 (syst), which is $2.1 \sigma$ larger than the value expected in the Standard Model. ${ }^{10}$

Recently, LHCb published a measurement of $R_{D^{*}}$ where $\tau$ has been reconstructed in three-prong decay $\tau^{+} \rightarrow \pi^{+} \pi^{-} \pi^{0} \bar{\nu}_{\tau}$ using the Run 1 dataset. ${ }^{11}$ The inclusion of charge-conjugate modes is implied throughout the document. The $D^{*}$ meson is reconstructed through the $D^{*-} \rightarrow \bar{D}^{0}\left(\rightarrow K^{+} \pi^{-}\right) \pi^{-}$decay chain. The visible final state consists of six charged tracks; neutral pions are ignored in this analysis. The signal yield is normalized to the exclusive $B^{0} \rightarrow D^{*-} \pi^{+} \pi^{-} \pi^{+}$decay channel which branching fraction has been measured by the B-factories. The similar topology between the signal and the normalization channel, results in large cancellations of the experimental uncertainties.

The analysis extracts the ratio $K\left(D^{*}\right)$ defined as:

$$
K\left(D^{*}\right)=\frac{\mathcal{B}\left(B^{0} \rightarrow D^{*-} \tau^{+} \nu_{\tau}\right)}{\mathcal{B}\left(B^{0} \rightarrow D^{*-} 3 \pi\right)}=\frac{N_{B^{0} \rightarrow D^{*-} \tau^{+} \nu_{\tau}}}{N_{B^{0} \rightarrow D^{*-} 3 \pi}} \frac{\epsilon_{B^{0} \rightarrow D^{*-} 3 \pi}}{\epsilon_{B^{0} \rightarrow D^{*-} \tau^{+} \nu_{\tau}}} \frac{1}{\mathcal{B}\left(\tau^{+} \rightarrow 3 \pi\left(\pi^{0}\right) \bar{\nu}_{\tau}\right)}
$$

where $N_{i}$ and $\epsilon_{i}$ are the yield and the selection efficiency respectively.

$R_{D^{*}}$ ratio is subsequently obtained by:

$$
R_{D^{*}}=K\left(D^{*}\right) \times \frac{\mathcal{B}\left(B^{0} \rightarrow D^{*-} 3 \pi\right)}{\mathcal{B}\left(\mathcal{B}\left(B^{0} \rightarrow D^{*-} \mu^{+} \nu_{\mu}\right)\right.}
$$

where the ratio on the right comes from external inputs. ${ }^{13}$

The main background contribution is due to the inclusive decays of b-hadron $H_{b} \rightarrow D^{*} 3 \pi X$. A good suppression of such background is obtained by requiring the $\tau$ vertex to be downstream with respect to $\mathrm{B}$ vertex along beam direction with a $4 \sigma$ significance. This requirement, applied along with other topological cuts, suppresses the dominant background by three order of magnitude. The background surviving the first selection is mainly due to double-charmed B decays of type $B \rightarrow D^{*-} D_{s}^{+}(X)$. In order to discriminate this background from signal, a multivariate algorithm (BDT) has been built based on isolation variables (describing how much the signal tracks are isolated from the other ones and using information derived by the neutral energy deposited in the events), on variables which exploit the differences between the resonant structures of the 3-pions system from signal and $D_{s}$ background decays and on kinematic variables computed under signal and background hypothesis.

The output of this algorithm has been fitted to extract the searched yields. Other variables which provide a good discrimination between signal and background are the $\tau$ decay time and the squared $B-D^{*}$ transferred momentum $q^{2}$. These variables depend on the $\mathrm{B}$ and $\tau$ momentum, but due to the presence of neutrinos in final states they can be determined up to a two-fold ambiguity. This problem is solved for both the $\tau$ and the B decay exploiting the excellent vertex capability of the $\mathrm{LHCb}$ vertex detector, which allows to determine with high precision the flight direction of the $\tau$ candidates and the B meson. 
To select normalization events, the $\tau$ vertex is required to be upstream with respect to $D^{0}$ vertex with a $4 \sigma$ significance. The yield of the normalization mode is determined by fitting the invariant mass of $D^{*-} 3 \pi$ system.

The signal yield is obtained from a three-dimensional extended maximum likelihood template fit on the output of the multivariate algorithm (in high BDT region), on the $\tau$ decay time and on $q^{2}$. The results of the fit are shown in Fig. 2. The shapes of the various components that contribute to the selected sample, are extracted from simulated events properly corrected according to various data control samples.

The measured $K\left(D^{*}\right)$ is:

$$
K\left(D^{*}\right)=1.93 \pm 0.13(\text { stat }) \pm 0.18(\text { syst })
$$

and using external inputs from PDG, the $R_{D^{*}}$ ratio is measured to be:

$$
R_{D^{*}}=0.285 \pm 0.019 \text { (stat) } \pm 0.025 \text { (syst) } \pm 0.013 \text { (ext) }
$$

where the third uncertainty originates from the limited knowledge of branching fraction of the normalization channel. This measure of $R_{D^{*}}$ is one of the most precise and is affected by the smallest statistical error. It is compatible with the SM calculation within one standard deviation.

The average of the two $R_{D^{*}}$ measurements here presented results $2.1 \sigma$ above the Standard Model predictions and(left) shows the current status of these measurements. Finally Fig. 2 shows also the HFLAV ${ }^{12}$ average of $R_{D^{*}}$ and $R_{D}$ measurement which result $4.1 \sigma$ from the SM expectations.
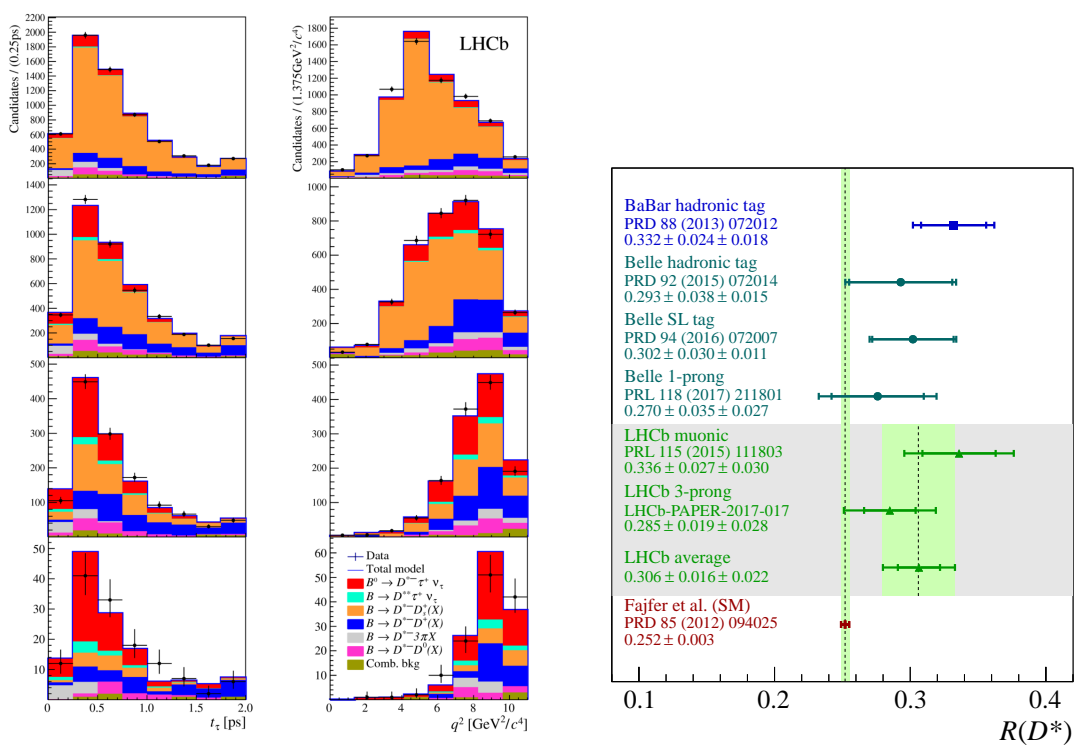

Fig. 2. (left) Distributions of (left) $t_{\tau}$ and (right) $q^{2}$ in four different BDT bins, with increasing values of the BDT response from top to bottom. (right) Comparison of the $R_{D^{*}}$ with the previous results from the B-factories and with SM expectations. 


\section{A. Lupato}

\section{Conclusion}

This article presents a review of the Lepton Flavour Universality tests performed by the $\mathrm{LHCb}$ collaboration using rare and semileptonic decays on the 2011 and $2012 \mathrm{LHCb}$ data, and in particular the recent measurements of $R_{K^{* 0}}$ and of $R_{D^{*}}$ using three-prong $\tau$ decays. $R_{K^{* 0}}$ deviates from the SM by 2.1-2.3 (low bin) and $2.4-2.5 \sigma$ (central bin), while the LHCb average of $R_{D^{*}}$ is about $2 \sigma$ above the SM prediction. It is dominated by statistical error and therefore Run 2 LHC data will allow to reduce this uncertainty. The current status motivates further work both on the theory as well as on the experimental side to clarify the present observations.

\section{References}

1. Minoru Tanaka, Z.Physics C67, 321 (1995).

2. Damir Beirevi, Svjetlana Fajfer, Nejc Konik, Olcyr SumensariPhys. Rev. D 94, 115921 (2016).

3. A.Crivellin, D.Muller and T.Ota, JHEP 09, 040 (2017).

4. Wolfgang Altmannshofer, Stefania Gori, Stefano Profumo, Farinaldo S. Queiroz, JHEP 1612, 106 (2016).

5. Andrzej J. Buras, Fulvia De Fazio, Jennifer Girrbach, Maria V. Carlucci, JHEP 02, $023(2013)$.

6. Marzia Bordone, Gino Isidori, Andrea Pattori, Euro. Phys. J. C 76, 440 (2016).

7. Christoph Bobeth, Gudrun Hiller, Giorgi Piranishvili, JHEP 0712, 040 (2007).

8. LHCb Collab. (R.Aaijet al.), Phys. Rev. Lett 113, 151601 (2014).

9. LHCb Collab. (R.Aaijet al.), JHEP 08, 055 (2017).

10. LHCb Collab. (R.Aaijet al.), Phys. Rev. Lett 115, 111803 (2015).

11. LHCb Collab. (R.Aaijet al.), ArXiv:1708.08856 (2017).

12. HFLAV, http://www.slac.stanford.edu/xorg/hfag/

13. Particle Data Group (C.Patrignaniet al.) Chin. Phys. C 40, 100001 (2016). 